\title{
Structure-dependent Conducting Properties of Phosphonated Polypeptoid Electrolyte Membranes Revealed by Cryogenic Electron Tomography
}

\author{
Xi Jiang ${ }^{1 *}$, Jing Sun ${ }^{2}$, Ronald N. Zuckermann ${ }^{3}$ and Nitash P. Balsara ${ }^{1,4}$ \\ 1. Materials Sciences Division, Lawrence Berkeley National Laboratory, Berkeley, CA 94720, USA \\ 2. Key Laboratory of Biobased Polymer Materials, Shandong Provincial Education Department; School \\ of Polymer Science and Engineering, Qingdao University of Science and Technology, Qingdao, China. \\ 3. Molecular Foundry, Lawrence Berkeley National Laboratory, Berkeley, CA 94720, USA \\ 4. Department of Chemical and Biomolecular Engineering, University of California, Berkeley, CA \\ 94720, USA \\ * Corresponding author: xijiang@lbl.gov
}

Polymers that conduct protons in the hydrated state are of crucial importance in a wide variety of clean energy applications such as hydrogen fuel cells and artificial photosynthesis. Phosphonated and sulfonated polymers are known to conduct protons at low water content. One of the most widely studied sulfonated polymer electrolytes is Nafion. The structure of Nafion has been intensively studied using scattering methods [1, 2] and electron tomography[3],[4]. Polymer electrolyte membranes are exposed to air, and thus the extent of hydration of the membrane is determined by the partitioning of water between the membrane and the surrounding gas phase. As a result, sulfonic acid-based membranes are ineffective proton transporters at high temperatures. Phosphonated electrolytes can release two protons instead of one and show higher chemical and thermal stability as compared to sulfonated electrolytes. In the previous work[5], we demonstrated that proton conductivity was maximized in high molecular weight, symmetric phosphonated polypeptoid electrolytes. However, the relationship between the structure of ion channels and conductivity has not yet been revealed in the hydrated membranes.

In this study, we use cryogenic electron tomography (cryo-ET) to characterize the presence of the ion channels in the dry and hydrated phosohonated popypeotoid membranes respectively. Polypeptoids are a family of comb-like polymers based on an N-substituted glycine backbone with precisely controlled chain length and compositions. We have synthesized two phosphonated polypeptoid diblock copolymers, poly-N-(2-ethyl)hexylglycine-block-poly-N-phosphonomethylglycine (pNeh- $b$-pNpm), with same block ratio but different chain length. The polydispersity (PDI) is about 1.0003. The chemical structures of two polypeptoids diblocks copolymer are shown in Figure 1A. The ion exchange capacity (IEC) of polypeptoids are $3.06 \mathrm{mmol} / \mathrm{g}\left(\mathrm{pNeh}_{9}-b-\mathrm{pNpm}_{9}\right)$ and $3.09 \mathrm{mmol} / \mathrm{g}\left(\mathrm{pNeh}_{18}-b-\mathrm{pNpm}_{18}\right)$ respectively. In spite of the similarity in IEC, the conductivity of $\mathrm{pNeh}_{18}-b-\mathrm{pNpm}_{18}(8.1 \mathrm{mS} / \mathrm{cm})$ is about 4 times higher than the conductivity of $\mathrm{pNeh}_{9}-b-\mathrm{pNpm}_{9}(1.9 \mathrm{mS} / \mathrm{cm})$ measured in the hydrated membranes. Figure 1B and 1C show the slices of tomograms obtained from the drop casted dry membranes which were annealed in humid chamber with $\mathrm{RH}=98 \%$ (the relative humidity at which proton conductivity was measured). The dark lamellar structure can be observed in both dry and hydrated films. It can be attributed to the conductive phosphonated blocks. Figure 1D and 1E show the slices of tomograms obtained from the vitrified hydrated membranes. It is evident that the $\mathrm{pNeh}_{18}-b$ $\mathrm{pNpm}_{18}$ membrane shows the honeycomb structure comprising the conductive phosphonated blocks. It is fundamentally different from the lamellar structure of the $\mathrm{pNeh}_{9}-b-\mathrm{pNpm}_{9}$ membrane. It can be concluded that the honeycomb-like percolating hydrophilic channels comprising phosphonated blocks increases the proton conductivity significantly as compared to the randomly interconnected lamellar channels in the hydrated membranes. 
References:

[1] G Gebel and J Lambard, Macromolecules 30 (1997), p. 7914.

[2] MA Modestino et al., Macromolecules 46 (2013), p. 867.

[3] S Yakovlev et al., J Am Chem Soc 133 (2011), p. 20700.

[4] FI Allen et al., Acs Macro Lett 4 (2015), p. 1.

[5] J Sun et al., Macromolecules 49 (2016), p. 3083.

[6] Funding for this work was provided by the Soft Matter Electron Microscopy Program (KC11BN), supported by the Office of Science, Office of Basic Energy Science, US Department of Energy, under Contract DE-AC02-05CH11231. Work at the Molecular Foundry was supported by the Office of Science, Office of Basic Energy Sciences, of the U.S. Department of Energy under Contract No. DEAC02-05CH11231. Micrographs presented here were obtained at the Donner Cryo-EM facility in Lawrence Berkeley National Lab.

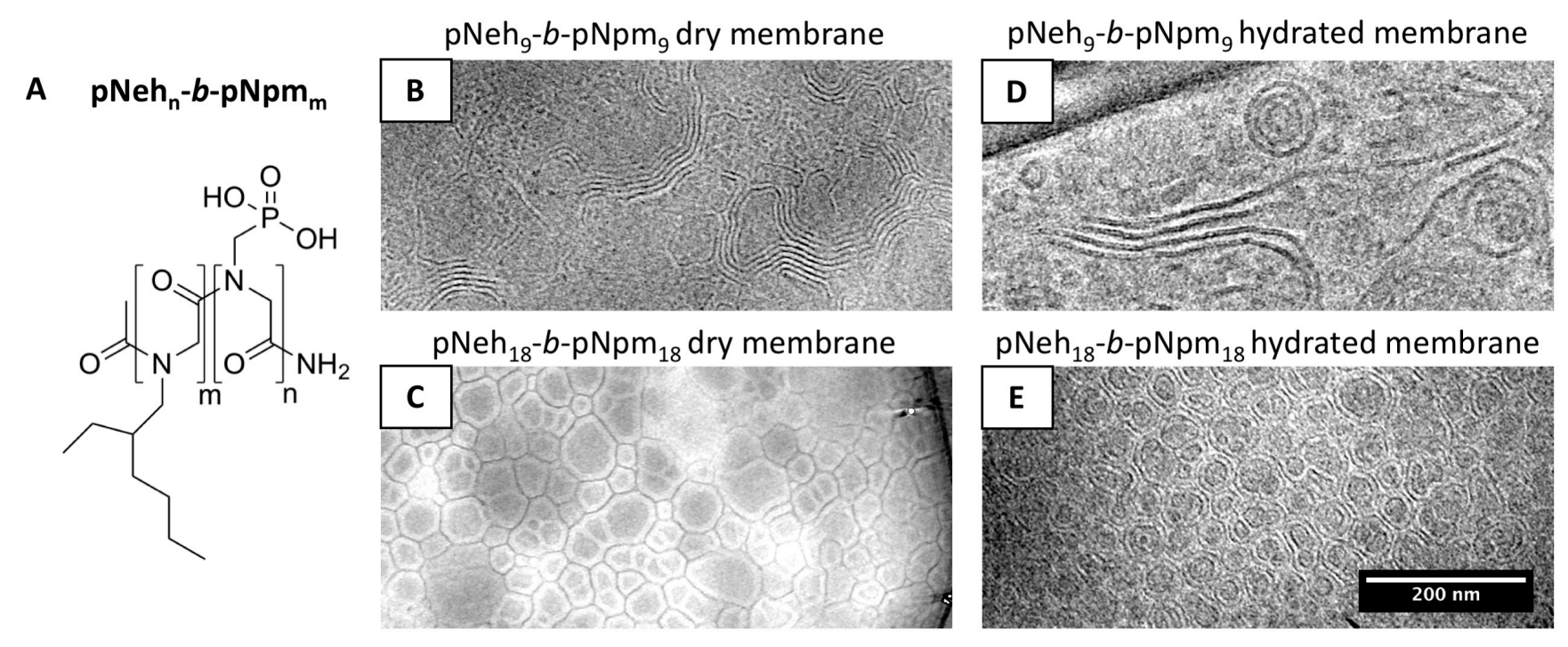

Figure 1. A. Chemical structure of $\mathrm{pNeh}_{\mathrm{n}}-b-\mathrm{pNpm}_{\mathrm{m}}$ polyepeptoids. B. Slice of tomogram obtained from annealed dry $\mathrm{pNeh}_{9}-b-\mathrm{pNpm}_{9}$ free standing thin film. C. Slice of tomogram obtained from annealed dry $\mathrm{pNeh}_{18}-b-\mathrm{pNpm}_{18}$ free standing thin film. D. Slice of tomogram obtained from hydrated $\mathrm{pNeh}_{9}-b-\mathrm{pNpm}{ }_{9}$ free standing thin film. E. Slice of tomogram obtained from hydrated $\mathrm{pNeh}_{18}-b-\mathrm{pNpm}_{18}$ free standing thin film. Scale bar applies to all images. 